\title{
Percepção de enfermeiros da rede cegonha sobre sinais do início do trabalho de parto
}

\author{
Perception of nurses from the maternal-child health services about signs of the beginning of \\ childbirth work \\ Percepción de enfermeros de los servicios de salud materno-infantil sobre señales del inicio del \\ trabajo de parto
}

Recebido: 19/03/2021 | Revisado: 08/04/2021 |Aceito: 17/04/2021 | Publicado: 01/05/2021
Ivelise Fhrideraid Alves Furtado da Costa ORCID: https://orcid.org/0000-0001-5455-3266 Universidade de Pernambuco, Brasil E-mail: ivelisefurtado@gmail.com
Francisco Stélio de Sousa ORCID: https://orcid.org/0000-0002-9604-4133 Universidade Estadual da Paraíba, Brasil E-mail: stelio_uepb@yahoo.com.br
Adriana Gomes Nogueira Ferreira ORCID: https://orcid.org/0000-0002-7107-1151 Universidade Federal do Maranhão, Brasil E-mail: adrianagn2@hotmail.com
Fernanda Albuquerque de Oliveira ORCID: https://orcid.org/0000-0003-0541-0351 Universidade Estadual da Paraíba, Brasil E-mail: nanda-oi@hotmail.com
Ana Raquel Silva Souza
ORCID: https://orcid.org/0000-0002-4919-5705 Universidade Estadual da Paraíba, Brasil E-mail: anaraquel.coracao2@gmail.com
Tatiane Gomes Guedes
ORCID: https://orcid.org/0000-0001-7149-2290 Universidade Federal de Pernambuco, Brasil E-mail: tatigguedes@yahoo.com.br Inácia Sátiro Xavier de França ORCID: https://orcid.org/0000-0002-2695-510X Universidade Estadual da Paraíba, Brasil E-mail: inacia.satiro@gmail.com Rosilene Santos Baptista ORCID: https://orcid.org/0000-0001-7400-7967 Universidade Estadual da Paraíba, Brasil E-mail: rosilenesbaptista@gmail.com

\begin{abstract}
Resumo
Objetivos: Identificar os sinais de início de trabalho de parto, segundo os profissionais da rede cegonha. Metodologia: Trata-se de um estudo transversal, com abordagem de dados primários, realizado em unidades da Rede Cegonha, no período de agosto de 2017 a julho de 2019. A amostragem foi de 84 enfermeiros. A coleta de dados foi efetuada por formulário contendo variáveis de interesse do estudo. Efetuou-se estatística descritiva, para toda a estatística adotouse o nível de significância de 5\%. Esta pesquisa foi aprovada sob-registro CAAE: 87854418.3.0000.5187.Resultados: Quanto aos sinais de início do trabalho de parto foram citados: saída do tampão mucoso, contrações que aumentam sua força constantemente e dor que inicia na região lombar e migra para o púbis $(47,94 \%$, $n=35)$.Também foram citadas: contrações uterinas com padrão diferente das recomendações vigentes e perdas vaginais $(52,63 \%, \mathrm{n}=40)$; contrações, perdas vaginais e dilatação $(10,52 \%, n=8)$; só contrações $(15,76 \%, n=12)$. Conclusões: Não há apenas um sinal ou sintoma que seja definitivo para identificação do início de trabalho de parto, mas que deve-se levar em consideração a subjetividade e multifatoriedade que envolve o atendimento, reconhecendo cada sinal e sintoma. Sendo necessário o aperfeiçoamento do preparo para o parto a fim de colaborar para uma melhor assistência.

Palavras-chave: Serviços de saúde materno-infantil; Acolhimento; Início do trabalho de parto; Trabalho de parto.
\end{abstract}

\section{Abstract}

Objectives: To identify the signs of labor onset, according to the professionals of the Cegonha network. Methodology: This is a cross-sectional study, with a primary data approach, carried out in units of the Rede Cegonha, from August 
2017 to July 2019. The sample consisted of 84 nurses. Data collection was performed using variables of interest to the study. A descriptive statistic was performed, for all statistics the significance level of 5\% was adopted. This research was approved under CAAE registration: 87854418.3.0000.5187. Results: regarding the signs of the beginning of labor, the following were mentioned: exit of the mucous plug, contractions that constantly increase its strength and pain that starts in the lumbar region and migrates to the pubis $(47.94 \%, \mathrm{n}=35)$. Also cited were: uterine contractions with a different pattern from the current recommendations and vaginal losses $(52.63 \%, \mathrm{n}=40)$; contractions, vaginal losses and dilation $(10.52 \%, \mathrm{n}=8)$; contractions only $(15.76 \%, \mathrm{n}=12)$. Conclusions: There is not only one sign or symptom that is definitive for the identification of the beginning of labor, but one must take into account the subjectivity and the multifactorial nature that involves care, recognizing each sign and symptom. It is necessary to improve the preparation for childbirth in order to collaborate for a better assistance.

Keywords: Maternal-Child health services; User embracement; Labor onset; Labor, Obstetric.

\section{Resumen}

Objetivos: identificar las señales de inicio de trabajo de parto, según los profesionales de la Red Cegonha. Metodología: se trata de un estudio transversal, con enfoque de datos primarios, realizado en unidades de la Red Cegonha, en el período de agosto de 2017 a julio de 2019. El muestreo fue de 84 enfermeros. La recopilación de datos fue realizada por formulario conteniendo variables de interés del estudio. Se efectuó estadística descriptiva, para toda la estadística se adoptó el nivel de significación de 5\%. Esta investigación fue aprobada bajo registro CAAE: 87854418.3.0000.5187. Resultados: en cuanto a las señales de inicio del trabajo de parto fueron citados: salida del tapón mucoso, contracciones que aumentan su fuerza constantemente y el dolor que inicia en la región lumbar y sigue para el pubis $(47,94 \%, n=35)$. También fueron citadas: contracciones uterinas con estándar diferente de las recomendaciones vigentes y pierdas vaginales $(52,63 \%, \mathrm{n}=40)$; contracciones, pierdas vaginales y dilatación $(10,52 \%, n=8)$; solo contracciones $(15,76 \%, n=12)$. Conclusiones: no solo hay una señal o un síntoma que sea definitivo para la identificación del inicio del trabajo de parto, pero se debe llevar en consideración la subjetividad y los varios factores que implican el servicio, reconociendo cada señal y síntoma. Siendo preciso el perfeccionamiento de la preparación para el parto a fin de colaborar para una mejor asistencia.

Palabras clave: Servicios de salud materno-infantil; Acogimiento; Inicio del trabajo de parto; Trabajo de parto.

\section{Introdução}

O período gestacional e o trabalho de parto ainda são interpretados como uma ameaça à saúde materno-infantil (Mahalingam \& Venkateasan, 2017), panorama, sobretudo fundamentado na incidência de morbimortalidade maternas,(36\%) e Razão de Morte Materna (RMM) 66.6/100000 Nascidos Vivos (NV), respectivamente-, na prevalência do uso de indutores e analgésicos -67,9\%, assim como na ocorrência de partos cirúrgicos -49\% - (Ministério da Saúde, 2013; 2016; Zhao et al., 2017).

Acentuando tal cenário, mantém-se a fragilidade do sistema de referência entre a atenção básica e a média complexidade, responsável pela assistência ao Trabalho de Parto (TP); que se atrela também à medicalização abusiva da parturição e à alta frequência de partos pré-termos - 46\% - (Ministério da Saúde, 2016; Victora et al., 2011). Especialmente na região Nordeste, é significativa a incidência de óbitos maternos (RMM: 95/100.000 NV) e de parto cesariano (59\% das primíparas) (Morse et al., 2011; Dias et al., 2016).

Tal panorama segue os padrões internacionais de mortalidade materna com 303000 mortes maternas em 2015, assim como o aumento exponencial da incidência de cesarianas em cerca de 121 países analisados (Zhao et al., 2017), a América Latina, em especial, teve a maior incidência $(40,5 \%)$ e o maior incremento $(19,4 \%)$ em 24 anos, sendo registrado, no Brasil, o maior índice da região $(55,6 \%)$ (Bétran et al., 2016).

As revisões sistemáticas de Hanada e colaboradoes (2015) e Kobayashi e colaboradores (2017) atestam que, como descrito, tal problema está relacionado com a admissão e intervenções inadequadas, durante a fase latente, e mesmo na ativa do TP. Há relatos, no entanto, de que a admissão no TP ativo reduziu o uso de analgesia epidural e o prolongamento do TP, resultando em maior satisfação materna (Kobayashi et al., 2017).

Neste sentido, o Ministério da Saúde preconiza o acolhimento a gestantes por meio da Rede Cegonha, tal acolhimento cursa com a classificação de risco, recepcionando as gestantes que chegam com sinais de trabalho de parto ativo ou latente. 
Direcionado-as para profissionais especializados, conforme a complexidade dos sinais, sintomas e queixas; obedecendo fluxogramas, dando prioridade ao atendimento àquelas em situações mais grave e orientando as não prioritárias (Ministério da Saúde, 2014; 2014).

No entanto, foi constatado que tal acolhimento e a classificação de risco obstétrico têm sido ineficazes (Paisley; Wallace; Durant; 2011), apesar de sua potencialidade em evitar intervenções desnecessárias (Meirowitz et al., 2014); havendo o agravante de profissionais e estudantes demonstrarem pouco conhecimento a respeito do seu decurso e crerem que, em sua maioria, se fazem necessárias intervenções para sua conclusão (Shub et al., 2012).

O Ministério da Saúde define que os principais sinais de alerta, em relação ao TP, durante o período gestacional, são: sangramentos vaginais, contrações irregulares e perda de líquido; na ausência destes, a fase latente do parto se inicia com a percepção das contrações de Braxton-Hicks e a saída do tampão mucoso, evoluindo para o endurecimento do baixo ventre durante trinta segundos por intervalos de cinco minutos durante a primeira hora, podendo se postergar durante oito a doze horas (Ministério da Saúde, 2016).

Para além das definições clássicas, os sinais de início do TP são difíceis de determinar prospectivamente (Ministério da Saúde, 2016), são inespecíficos e subjetivos, ademais as recomendações para identificação do início de TP são versáteis, porém, são imprescindíveis ao encadeamento seguro do TP verdadeiro, uma vez que admitir uma gestante que não está em TP verdadeiro ou não orientar, ou não intervir da forma adequada trará repercussões negativas para mãe e feto (Pates, Mcintire \& Leveno, 2007; Green \& Spybi, 2009; How, Khoury \& Sibai, 2009; Harper et al., 2014; Henderson \& Redshaw, 2017; Østborg, Ronundstad \& Eggebø 2017).

Determinar o momento adequado para assistência ao parto normal previne resultados desfavoráveis para a mulher parto sem assistência profissional, morbidade e mortalidade materna -, no entanto, a assistência precoce promove intervenções desnecessárias como o uso de ocitocina, de analgesia e da cesariana, além da parada da progressão do parto, bem como maior tempo de TP e maior incidência de infecção puerperal (Ministério da Saúde, 2016).

A prolongada fase latente do TP apresenta relação com uso de ocitocina, cesariana, presença de mecônio no líquido amniótico (Henderson \& Redshaw, 2017; Bor et al., 2016), Apgar no 5 min inferior a 7, necessidades de manobras de ressuscitação e internamento em unidade de terapia intensiva neonatal. Logo é de extrema relevância que ambas as fases sejam delineadas, visando capacitar a gestante para o autodiagnostico e subsequente busca de assistência profissional (Hanley et al., 2016).

Diante desse panorama, se faz necessário avaliar o conhecimento sobre os sinais de início de trabalho de parto dos enfermeiros que realizam o acompanhamento pré-natal e o acolhimento obstétrico na rede cegonha.

\section{Metodologia}

\section{Tipo de estudo}

Compreende um estudo epidemiológico observacional retrospectivo de corte transversal, com abordagem quantitativa de dados primários. A pesquisa descritiva tem por finalidade observar, descrever e investigar aspectos de uma situação. O delineamento avaliativo constitui uma investigação elaborada para desvendar o funcionamento de um tratamento, política, prática ou programa. Tendo como valor primordial sua capacidade de adquirir respostas a questões práticas, reportadas pelas pessoas que necessitam chegar a uma decisão (Polit \& Beck; 2011).

\section{Local do estudo e período de realização}

O estudo foi realizado em 66 Unidades Básicas da Estratégia Saúde da Família do Município de Campina Grande, PB, Brasil. O período de coleta cursou entre novembro de 2018 a março de 2020. 


\section{População e amostragem}

A Estratégia Saúde da Família do Município de Campina Grande compreende 10 distritos sanitários, com 79 unidades básicas de saúde urbanas, com equipe de 100 enfermeiros, dentre os quais 81 foram entrevistados, tendo sido a previsão inicial de 80 profissionais, com base no cálculo amostral para população finita abaixo, com nível de significância de 5\%.

$$
\mathrm{n}=\frac{\mathrm{N} \cdot \sigma^{2} \mathrm{z}_{\gamma / 2}^{2}}{(\mathrm{~N}-1) \cdot \varepsilon^{2}+\sigma^{2} \mathrm{z}_{\gamma / 2}^{2}}
$$

Onde: $\mathrm{N}$ é o tamanho da população; $\sigma$ é o desvio padrão populacional; $\varepsilon$ é o erro amostral (5\%); $z^{2} \gamma^{2}$ é o grau de confiança obtido da tabela normal padrão.

\section{Critérios de elegibilidade}

Inclusão:

Enfermeiros da atenção básica que realizaram assistência pré-natal há um período superior a 12 meses.

Exclusão:

Todos os enfermeiros que não efetuem o preparo para o parto, nem visita puerperal rotineiramente.

\section{Variáveis estudadas}

Variáveis demográficas e socioeconômicas:idade, sexo, cor, estado conjugal, classe econômica, formação profissional, ocupação.

Variáveis relacionadas com o preparo para o parto: preparo para o parto, participação do companheiro no pré-natal, escolha do local do parto.

Variáveis relacionadas com o risco obstétrico: sinais do início do trabalho de parto, classificação de risco obstétrico.

\section{Procedimentos para a coleta de dados}

Os enfermeiros foram serão convidados para participarem voluntariamente do estudo a partir da apresentação da sua importância para o aperfeiçoamento da assistência. Após o consentimento, por meio de formulário (APÊNDICE A), foi efetuado o levantamento dos conhecimentos e práticas adotadas no preparo para o parto (sinais do início do TP verdadeiro) durante o pré-natal de risco habitual, conforme as recomendações do American College of Obstetricians and Gynecologists (2011; 2017) e do Ministério da Saúde (2016; 2017; 2014).

\section{Processamento e análise dos dados}

O formulário foi duplamente digitado e submetido à validação no subprograma Validate do Epi Info 5.3.2, utilizado, juntamente com o Epi Info 7.0 e R para o processamento das análises estatísticas.

Foi efetuada estatística descritiva por meio das medidas de tendência central para as variáveis numéricas e medidas de frequência absoluta e relativa para as variáveis categóricas. Para toda a estatística foi adotado o nível de significância de 5\%. Foi testada a distribuição de normalidade através do teste de Kolmogorov-Smirnov.

Para a avaliação dos formulários foram realizadas análises de variância, Teste-t, Qui-quadrado e teste exato de Fisher a fim de detectar o efeito do conhecimento dos sinais de início do TP verdadeiro sobre os desfechos gestacionais maternos, e a experiência e satisfação com o parto.

As variáveis que não atenderam aos pressupostos de normalidade da distribuição, requeridos pela ANOVA e teste-t, foram analisadas por meio d os correspondentes não-paramétricos Kruskall-Wallis e Wilcoxon-Mann-Whitney. Na análise de 
variância e teste-t, cada profissional foi considerado como uma réplica na estatística. Na ausência de réplica de tratamentos foram utilizados os testes de qui-quadrado e exato de Fisher, que consistem de análises tabulares da contagem de cada critério pelo total de cada perfil do profissional. Para toda a estatística foi adotado o nível de significância de 5\%.

\section{Aspectos éticos da pesquisa}

Tem sido respeitada a Resolução 466/2012 do Conselho Nacional de Saúde; onde o projeto foi submetido pela Plataforma Brasil a apreciação do Comitê de Ética e Pesquisa do Hospital Universitário Alcides Carneiro (HUAC), após a adequada autorização institucional, tendo sido aprovado sob registro CAAE: 92973018.0.0000.5175. Também, neste estudo todos os participantes assinarão o Processo de Consentimento Livre e Esclarecido (PCLE).

\section{Resultados}

Foram abordados 91 enfermeiros, dentre eles um foi excluído pelo tempo de experiência inferior a 12 meses, houveram quatro recusas, quatro licenças médicas e uma ausência, resultando em uma amostragem de 84 profissionais. A caracterização da amostra encontra-se compilada na tabela 1.

Dentre os entrevistados 95,24\% ( $\mathrm{n}=80$ ) são do sexo feminino, com média de idade de 42 anos, tendo 26 anos de idade o mais jovem e 66 o mais velho, com moda de 38 anos. Em sua maioria casados $(58,33 \%$, $n=49)$ e cuja cor da pele mais prevalente era a parda $(58,33 \%, \mathrm{n}=49)$. Quanto à classe socioeconômica 36,9\% $(\mathrm{n}=31)$ pertenciam a classe $\mathrm{B} 1$, tendo por renda aproximada $\mathrm{R} \$ 2.965,69$.

No que se refere à formação profissional, 58,33\% (n=49) dos enfermeiros se formaram há mais de 15 anos e 41,67\% $(\mathrm{n}=35)$ há menos de 15 anos, respectivamente. Em sua maioria obtiveram o título em instituição pública (69,04\%, n=58), sendo apenas $60,71 \%(\mathrm{n}=51)$ licenciados. Dentre os profissionais 13,10\% $(\mathrm{n}=11)$ concluíram outra graduação, porém apenas dois o fizeram na área de saúde - educação física e ciências biológicas.

A pós-graduação Latu sensu foi prevalente $(83,33 \%, n=70)$ entre os profissionais, dentre as especializações obtidas 78,87\% (n=56) foram em saúde da família, seguido de 21,13\% (n=15) de outras áreas, incluindo 8,33\% (n=7) de especialistas em obstetrícia. Já a pós-graduação Strictu sensu foi efetuada por 20,24\% (n=17) dos profissionais, havendo apenas um na área de enfermagem e os demais em saúde pública.

Tabela 1: Distribuição dos profissionais de acordo com as características socioeconômicas, demográficas e de formação profissional. Campina Grande, Paraíba, Brasil. 2019-2020.

\begin{tabular}{lcccc}
\hline \multicolumn{1}{c}{ Variável } & \multicolumn{2}{c}{ Masculino } & \multicolumn{2}{c}{ Feminino } \\
& $\mathrm{n}$ & $\%$ & $\mathrm{n}$ & $\%$ \\
\hline Estado civil (casado) & 3 & 6,12 & 46 & 93,88 \\
\hline Cor da pele (pardo) & 4 & 8,16 & 45 & 91,84 \\
\hline Classe socioeconômica (B1) & 2 & 6,45 & 29 & 93,55 \\
\hline Tempo de formação (>15 anos) & 2 & 4,08 & 47 & 95,92 \\
\hline Pós-graduação (saúde pública) & 2 & 3,57 & 54 & 96,43 \\
\hline Total & 4 & 4,76 & 80 & 95,24 \\
\hline
\end{tabular}

Fonte: autores.

Ao serem questionados a respeito de capacitações e aperfeiçoamentos realizados na área de atenção pré-natal 73,81\% (n=62) não efetuou nenhuma capacitação nos últimos nove anos, de maneira análoga 57,32\% ( $\mathrm{n}=55)$ dos enfermeiros entrevistados não possui aperfeiçoamento nessa área. 
Dentre os que efetuaram capacitação prevaleceram cursos dos últimos três anos, sendo que 13,25\% (n=11) o fizeram voltado exclusivamente para questões outras que não o parto, cuja carga horária média foi de 143,43 horas. Já dentre os que se aperfeiçoaram, 56,25\% ( $\mathrm{n}=45)$ o fizeram por meio de cursos de carga horária média de 10,75 horas, voltados para a temática do parto.

Os enfermeiros entrevistados apresentaram tempo médio de experiência na atenção básica de 13,88 anos (DP $\pm 7,54)$, moda e mediana de 14 anos. Quando questionados a respeito da experiência em outros níveis de atenção à saúde 75\% (n=63) afirmaram possuir, apresentando média de 6,29 anos ( $\mathrm{DP} \pm 6,43)$, havendo 24,2\% (n=15) desses com experiência em maternidade.

Tabela 2: Distribuição dos profissionais de acordo o sexo sem a formação voltada a atenção pré-natal. Campina Grande, Paraíba, Brasil. 2019-2020.

\begin{tabular}{lcccc}
\multicolumn{1}{c}{ Variável } & \multicolumn{2}{c}{ Masculino } & \multicolumn{2}{c}{ Feminino } \\
& $\mathrm{n}$ & $\%$ & $\mathrm{n}$ & $\%$ \\
\hline Sem capacitação & 3 & 4,84 & 59 & 95,16 \\
Sem aperfeçcamento & 2 & 5,71 & 33 & 94,29 \\
\hline
\end{tabular}

Fonte: autores.

Foi significativa a incidência de outro vínculo $(50 \%, n=42)$, sendo $15,66 \%(n=13)$ na média complexidade, 10,84\% $(n=9)$ na alta complexidade e 19,28\% $(n=16)$ em outras atividades destacando-se a preceptoria e à docência $(81,25 \%$, $n=13$; $18,75 \%, \mathrm{n}=3)$.

No que se refere aos sinais de início do trabalho de parto os entrevistados citaram a saída do tampão mucoso $(77,38 \%$, $\mathrm{n}=65)$, contrações que aumentam sua força constantemente $(58,33 \%, \mathrm{n}=49)$, dor que inicia na região lombar e migra para o púbis $(58,33 \%, \mathrm{n}=49)$, bem como a associação dos três sinais supracitados $(41,66 \%, \mathrm{n}=35)$. Desta forma, foi prevalente $(46,42 \%, n=39)$ a referência a outros sinais de início do trabalho de parto ativo. Ressalta-se que dentro os entrevistados cinco não responderam a esse questionamento.

Também foram citadas contrações uterinas com padrão diferente das recomendações do ministério da saúde e do colégio americano de ginecologistas e obstetras $(66,66 \%, \mathrm{n}=56)$, perdas vaginais $(63,09 \%$, $\mathrm{n}=53)$ e dilatação uterina $(18,60 \% \%, \mathrm{n}=15)$. Assim como a associação de tais eventos contrações e perdas vaginais $(50 \%$, $n=42)$; contrações, perdas vaginais e dilatação $(9,52 \%, n=8)$; só contrações $(14,28 \%, n=12)$. Dentre os entrevistados, apenas quatro não citaram outros sinais.

Tabela 3: Distribuição do conhecimento dos enfermeiros, que prestam assistência ao pré-natal de baixo risco, sobre os sinais de início do TP. Campina Grande, Paraíba, Brasil. 2019-2020.

\begin{tabular}{lcccc}
\hline \multicolumn{1}{c}{ Variável } & Masculino & \multicolumn{2}{c}{ Feminino } \\
& $\mathrm{N}$ & $\%$ & $\mathrm{n}$ & $\%$ \\
\hline $\begin{array}{l}\text { Sinais de trabalho de parto ativo } \\
\begin{array}{l}\text { Outros sinais de trabalho de } \\
\text { parto ativo }\end{array}\end{array}$ & 2 & 5,71 & 33 & 94,32 \\
\hline
\end{tabular}

Fonte: autores. 


\section{Discussão}

Foi encontrado, perfil etário profissional semelhante ao estudo de Baptista et. al. (2015), que abordou a mesma população. Analisando tal perfil observado, percebe-se a evolução quanto a formação profissional, com o incremento de pósgraduação Strictu sensu e Latu sensu em saúde da família comparada a estudo anterior (Baptista et. al., 2015).

Destaca-se a ausência de capacitações voltadas a assistência pré-natal, sendo um agravante o fato de o enfermeiro ser o profissional responsável pelo acompanhamento pré-natal de risco habitual até o terceiro trimestre gestacional (Ministério da Saúde, 2016). Sabendo que orientações pré-natais fomentam a confiança, o empoderamento e a capacidade de enfrentamento frente as peculiaridades inerentes ao trabalho de parto de primíparas (Henderson \& Redshaw, 2017).

Estudo recente atesta que gestantes não tem recebido orientações devidas, dos profissionais de saúde, acarretando na insegurança e na ausência de protagonismo (Oliveira et al., 2012). No decorrer de tal acompanhamento, as orientações quanto a fatores de risco, sinais de alerta, bem como sinais de início do trabalho de parto devem ser implementados durante o terceiro trimestre gestacional (Ministério da Saúde, 2013).

O preparo para o parto é considerado ferramenta essencial para a prevenção de intervenções desnecessárias e promoção de saúde e bem-estar, uma vez que o parto é um evento que se inicia por meio de um processo, que envolve alterações hormonais e respostas fisiológicas às mesmas, tal processo sofre influência de diversos elementos externos como nível socioeconômico e grau de instrução, além percepções e experiências (Oliveira et al., 2012).

Diante disso, as primigestas apresentam maior fragilidade diante do início do TP, tendo em vista a ineditude do evento, os personagens e as sensações envolvidas. Sendo comum buscarem apoio profissional precoce, uma vez que sentem segurança e confiança ao delegarem aos mesmos a identificação e confirmação do início do trabalho de parto (Eri et al.,2015).

O trabalho de parto, em si, é de difícil identificação devido as peculiaridades do ser humano (Marowitz, 2014; Gross et al., 2009). Mesmo que o caminho natural envolva a produção de hormônios, levando a coordenação das contrações uterinas e mudanças na cérvix que ocorrem antes do início do trabalho de parto ativo (Marowitz, 2014). O deficiente autocuidado, autoconhecimento e empoderamento afetam negativamente a capacidade de reconhecer os sinais de trabalho de parto ativo, agravando a insegurança e ansiedade (Oliveira et al., 2012).

Quanto aos sinais de trabalho de parto é sabido que em estudo realizado em Mossoró, as nulíparas referiram como sinais de trabalho de parto ativo perdas vaginais e contrações uterinas (Milfont et al., 2011).

Revisão constata que é difícil a determinação do início da fase latente do TP, assim como da fase ativa, porém estudos evidenciam maior prevalência de relatos de contrações uterinas regulares acompanhadas da dilatação progressiva do colo uterino, entretanto, não há evidencia científica que sustente a afirmação que tais sinais indicam de forma eficaz e imutável o início do TP (Henderson \& Redshaw, 2017).

Como se observa em estudos recentes em que sua definição varia desde sinais físicos (dilatação do colo entre 3 e 4 cm, progressão da dilatação em $1 \mathrm{~cm}$ por hora), a sinais clínicos - 10 a 12 contrações uterinas por hora, aumento de marcadores séricos (folistatina, prostaglandina) - (Pates, Mcintire \& Leveno 2007; How, Khoury \& Sibai, 2009 2009; Lee et al., 2008; Trae et al. 2007; Hoh et al., 2012; Neal et al., 2016). Tal incerteza gera respostas psicogênicas e fisiológicas maternas, refletindo negativamente na progressão do TP (Green \& Spybi, 2009).

Sabendo que o diagnóstico ineficaz do início do TP tem sido associado a maior probabilidade de distócias (Harper et al., 2014; Hoh et al., 2012), ao comprometimento do acompanhamento da sua progressão, agravando-se quando utilizados protocolos institucionais embasados na curva de Friedman (1978), sem correções (Hoh et al., 2012, Neal et al., 2016).

Corroborando com as informações supracitadas, estudo produzido na Inglaterra, com uma coorte de parturientes, evidenciou que ao buscarem os serviços de saúde as mesmas se encontravam em prolongamento da primeira fase do TP ativo, 
e devido a isso, necessitavam de intervenções como anestesia e cesariana (Gross et al., 2009). Para, além disso, na análise das intervenções realizadas, pesquisa experimental atestou que o uso de ocitocina prologa a fase ativa do TP (Østborg, Ronundstad \& Eggeb $\varnothing, 2017)$.

Enquanto experimento com uso de heparina de baixa densidade molecular, verificou o aumentou do risco de hemorragia e do parto pré-termo (Isma et al., 2010). Outro experimento atestou que a analgesia epidural autocontrolada esteve associada à alteração na progressão e na dilatação, durante a fase ativa do TP (Ye, 2015).

Em contrapartida, revisões atestam que não há critério estabelecido para o autodiagnostico de TP ativo, porém a sua determinação apresenta potencial para prevenir o diagnostico equivocado de distócias e assim intervenções desnecessárias, uma vez que, o TP ativo determinado de forma equivocada induz ao diagnóstico errôneo de distócia e, consequentemente, exporá mãe e feto a riscos. As dificuldades maternas em identificar o momento adequado de solicitar assistência ao parto geram ansiedade e insegurança, comprometendo a relação profissional-paciente (Lauzon \& Hodnett, 2009; Lauzon \& Hodnett, 2013).

Sendo evidentes as possíveis distócias, associadas ao diagnóstico ineficaz do início do TP (Harper et al., 2014; Hoh et al., 2012), percebe-se a necessidade de estabelecer um parâmetro confiável e estratégia eficaz de ensinamento, fomentando o empoderamento e o sentimento de controle, alterando significativamente a vulnerabilidade antes experimentada e, por conseguinte prevenindo os agravos associados.

O parto é um fenômeno natural inerente a existência humana, tendo sido medicalizado ao longo do tempo, pondo em cheque a capacidade feminina inata de dar à luz, mas não apenas isso, minou a confiança feminina para o autocuidado no decorrer deste fenômeno.

Diversos estudos atestam os malefícios das intervenções clínicas e cirúrgicas frente ao trabalho de parto, o qual muitas vezes não é avaliado de forma adequada. Visando dirimir tais prejuízos foi estabelecido uma rede de assistência à parturiente que inicia o seu acompanhamento para o parto ainda no pré-natal, por meio de acolhimento, de orientações para o parto, de visitas e de referências às maternidades; também foi instituído o acolhimento e classificação de risco obstétrico, o qual visa, entre outros, eliminar a admissão precoce e a peregrinação materna.

Porém para a determinação do início do trabalho de parto ativo, não há apenas um sinal ou sintoma que seja definitivo para sua identificação, pois cada organismo carrega peculiaridades que dificultam a padronização de sinais e sintomas, ademais também sofrem influencia de elementos externos.

Mesmo diante desses entraves, diversas organizações recomendam que o profissional deve atentar ao fisiológico; observando sinais clinicos de que o útero feminino está sob estímulo natural para o parto, por meio da presença de contrações ritmicas, regulares, apagamento e dilatação do colo uterino. Para tanto, é necessário qualificar adequadamente os envolvidos na assistencia ao pré-natal e parto.

Ademais, o autoconhecimento da parturiente e sua capaidade de autocuidado podem ser ferramentas uteis para melhor condução, levando em consideração a subjetividade e multifatoriedade que envolve o parto, reconhecendo cada sinal e sintoma. Sendo necessário o aperfeiçoamento do preparo para o parto a fim de colaborar para uma melhor assistência.

$\mathrm{O}$ estudo foi implementado em apenas uma cidade do interior do nordeste brasileiro, logo seus resultados têm sua abrangência limitada a esta região. Tal fato justifica-se pela ausência de financiamento, o que inviabiliza a expansão da coleta de dados para outras cidades e regiões.

Ademais o estudo abordou informações da prática profissional, porém requereu que os participantes citassem os sinais e sintomas que costumam observar, logo está sujeito a viés de memória.

Os resultados do estudo retratam uma realidade até então pouco estudada e enfatizada, tanto na prática quanto o ensino. Logo, voltar a atenção aos sinais de trabalho de parto ativo, acarretam no adequado preparo para parto, durante a 
gestação, e no alinhamento com todos os níveis de atenção, pondo em prática o sistema de referência e contrarreferência. Resultando no fortalecimento das ações da enfermagem na atenção da saúde da mulher durante o trabalho de parto e parto.

Diante do exposto, se faz necessário outros estudos que verifiquem o impacto das orientações sobre trabalho de parto sobre a autonomia e autocuidado da parturiente. Assim como, analisar amostra mais abrangente, tornando os resultados mais comparáveis a população brasileira.

\section{Agradecimentos}

Ao Laboratório de Análises Estatísticas (LANEST-UFCG), pela consultoria estatística de alta qualidade, em especial ao professor Dr. Gilberto Matos.

\section{Referências}

ACOG (2011). The American College of Obstetricians and Gynecologists. How to tell when labor begins. https://www.acog.org/Patients/FAQs/How-to-TellWhen-Labor-Begins

ACOG (2017). The American College of Obstetricians and Gynecologists. Approaches to Limit Intervention During Labor and Birth. Washigton: ACOG, $\mathrm{n}^{\circ} 687,9$ p. https://www.acog.org/Resources-And-Publications/Committee-Opinions/Committee-on-Obstetric-Practice/Approaches-to-Limit-InterventionDuring-Labor-and-Birth.

Baptista S R., et al. (2015). Assistência pré-natal: ações essenciais desenvolvidas pelos enfermeiros. Murcia: Enfermeira global,; (40): 112-127.

Bétran, A. P., Ye, J., Moller, A. B., Zhang, J., Gülmezoglu, A. M., \& Torloni, M. R. (2016). The Increasing Trend in Caesarean Section Rates: Global, Regional and National Estimates: 1990-2014. PLoS ONE 11(2): e0148343.

Bor, P., et al. (2016). Continuation versus discontinuation of oxytocin infusion during the active phase of labour: a randomised controlled trial. BJOG; $23: 129-$ 135.

Cheyne, H., Dowding, D. W., \& Hundley, V. (2006). Making the diagnosis of labour: midwives' diagnostic judgement and management decisions. Medford: Journal of Advanced Nursing, 53 (6): 625-635.

Dias, M. A. B., et al. (2016). Factors associated with cesarean delivery during labor in primiparous women assisted in the Brazilian Public Health System: data from a National Survey. Reproductive Health, 13, s. 3, (114): 175-185.

Eri,. T. S., et al. (2015). A balancing actin an unknown territory: A meta synthesis of first-time mothers' experiences in early labour. Amsterdam: Midwifery; 31 (3): e58-67.

Friedman, E. (1978). Labour: Clinical Evaluation and Management: Appleton Century Crofts, (2ª ed.,) 450 p.

Green, J. M., \&Spybi, H. (2009). Early labor: What's the problem? Medford: Birth, 34 (9): 332-339.

Gross, M., Burian, R. A., Fromke, K., Hecker, H., et al. (2009). Onset of labor: women's experiences and midwives' assessments in relation to first stage duration. Deutsche: Arch Gynecol Obstet, 280: 899-905.

Hanada, N. (2015). Psychosocial and educational interventions in latent phase or early labour for improving birth outcomes (Protocol). Cochrane Database of Systematic Reviews; 2 (CD011516).

Hanley, G. E., et al. (2016). Diagnosing onset of labor: a systematic review of definitions in the research literature. London: BMC Pregnancy and Childbirth, 16 (71): $11 \mathrm{p}$.

Harper, L. M., et al. (2014). Defining an abnormal first stage of labor based on maternal and neonatal outcomes. Philadelphia: Am J Obstet Gynecol, 210 (6): 536.e1-536.e7.

Henderson, J., \& Redshaw, M. (2017). Sociodemographic differences in women's experience of early labour care: a mixed methods study.: BMJ Open, 7 (e016351): 12p.

Hoh, J. K., et al. (2012). Estimating time to full uterine cervical dilation using genetic algorithm. Taiwan: Kaohsiung Journal of Medical Sciences, 28:423-428. How, H. Y., Khoury, J. C., \& Sibai, B. H. (2009). Cervical Dilatation on Presentation for Preterm Labor and Subsequent Preterm Birth. New York: Am J Perinatol, 2009; 26:1-6.

Isma, N., et al. (2010). The effect of low molecular weight heparin (dalteparin) on duration and initiation of labour. Netherlands: $J$ Thromb Thrombolysis, 30 (2):149-153.

Kobayashi, S., et al. (2017). Assessment and support during early labour for improving birth outcomes. Cochrane Database of Systematic Reviews; 4(CD011516): $62 \mathrm{p}$. 
Lauzon, L., \& Hodnett, E. D. (2009). Labour assessment programs to delay admission to labour wards. Ontario: Cochrane Database of Systematic Reviews, 2009; 3 (CD000936).

Lauzon, L., \& Hodnett, E. D. (2013). Antenatal education for self-diagnosis of the onset of active labour at term. Ontario: Cochrane Database of Systematic Reviews, 4 (CD000935).

Lee, S. E., et al. (2008) Amniotic fluid prostaglandin concentrations increase before the onset of spontaneous labor at term. United Kingdom: The Journal of Maternal-Fetal and Neonatal Medicine, 21 (2): 89-94.

Mahalingam, G., \& Venkateasan, M (2014). Mother's knowledge of warning signs of pregnancy, labour and puerperium.: Int J Med Sci Public Health; 3: 720722.

Marowitz, A. (2014). Caring for Women in Early Labor: Can We Delay Admission and Meet Women's Needs? : Journal of Midwifery \&Women's Health; 59 (6): 645-650.

Meirowitz, N., et al. (2014). Implementing an Obstetric Triage Acuity Tool in a High-Volume Obstetric Unit. Washington: Obstetrics \& gynecology.

Milfont, P. M. S., et. al.(2011). Exploratory study on the implementation of guidelines for a safe delivery and satisfaction of women. Niterói, RJ: Online Brazilian Journal of Nursing; 10 (3): 9p.

Ministério da Saúde, Secretaria de Atenção à Saúde, Departamento de Ações Programáticas Estratégicas (2013). Avaliação da atenção ao pré-natal, ao parto e aos menores de um ano na Amazônia Legal e no Nordeste, Brasil, 2010. Brasília: Ministério da Saúde; 1: $32-43$.

Ministério da Saúde, Secretaria de Atenção à Saúde, Departamento de Ações Programáticas Estratégicas (2013). Atenção ao pré-natal de baixo risco. Brasília: Ministério da Saúde; (1ª ed.) rev.: 46-49; 146-148.

Ministério da Saúde, Secretaria de Atenção à Saúde, Departamento de ações programáticas e estratégicas (2014). Caderneta da Gestante [Internet]. Brasília: Ministério da Saúde. 31 p.

Ministério da Saúde, Secretaria de Atenção à Saúde, Departamento de Ações Programáticas Estratégicas (2016). Protocolos da Atenção Básica: Saúde das Mulheres: Ministério da Saúde; 230 p.

Ministério da Saúde, Secretaria de Atenção à Saúde, Departamento de ações programáticas e estratégicas (2012). Caderneta da Gestante [Internet]: Ministério da Saúde; $31 \mathrm{p}$.

Ministério da Saúde, Secretaria de Atenção à Saúde, Departamento de Ações Programáticas Estratégicas (2017). Manual de Acolhimento e Classificação de $\begin{array}{llllllll}\text { Risco em Obstetrícia. Brasília: Ministério da } \quad \text { Saúde; } & \text { p.: } & 6-10 ; \quad 14-17 ; \quad 23 ; \quad 28 ; \quad 29\end{array}$ http://bvsms.saude.gov.br/bvs/publicacoes/manual_acolhimento_classificacao_risco_obstetricia_2017.pdf

Morse, N. L., et al. (2011). Mortalidade materna no Brasil: o que mostra a produção científica nos últimos 30 anos? Rio de Janeiro: Cad. Saúde Pública. 27 (4): 623-638.

Neal, J. L., et al. (2016). Pilot Study of Physiologic Partograph Use Among Low-Risk, Nulliparous Women With Spontaneous Labor Onset. Medford: $J$ Midwifery Womens Health; 61:235-241.

Oliveira, K. K. D., et al. (2012). Conception of Nulliparous on Labor Obstetric and Delivery.Rio de Janeiro:R. pesq.: cuid. fundam. online, 4(3): 2627-35. Østborg, T. B., Ronundstad, P.R., \& Eggeb $\emptyset$, T. M. (2017). Duration of the active phase of labor in spontaneous and induced labors. Copenhagen: Acta Obstet Gynecol Scand; 96(1): 120-127.

Paisley, K. S., Wallace, R., \& Durant, P. G. (2011). The Development of an Obstetric Triage Acuity Toolv. Bankock: MCN; 35 (15): $290-296$.

Pates, J. A., Mcintire, D. D., \& Leveno, K. J. (2007). Uterine Contractions Preceding Labor.New York: Obstetrics \& Gynecology; 110 (3): 566-569.

Polit, C. T., \& Beck, C. T. (2011) Fundamentos de pesquisa em enfermagem: Avaliação de evidências para a prática da enfermagem: Editora Artmed, (7): 263-287.

Shub, A., et al. (2012). Do primigravidae and their carers have a realistic expectation of uncomplicated labour and delivery? A survey of primigravidae in late pregnancy, obstetric staff and medical students. Australian and New Zealand Journal of Obstetrics and Gynaecology; 52: 73-77.

Trae, K., et al. (2007). Follistatin serum concentrations during full-term labour in women - significant differences between spontaneous and induced labour. Cambridge: Reproduction; 134: 705-711.

Victora, C. G., et al. (2011). Saúde de mães e crianças no Brasil: progressos e desafios. The Lancet; 32-46.

Ye, Y. (2015). Effects of Patient-Controlled Epidural Analgesia on Uterine Electromyography During Spontaneous Onset of Labor in Term Nulliparous Women. Thousand Oaks: Reproductive Sciences; 22 (11): 1350-1357.

Zhao, Y., Zhang, J., Zamora, J., et al. (2017). Increases in caesarean delivery rates and change of perinatal outcomes in low- and middle-income countries: a hospital-level analysis of two WHO surveys. Paediatr Perinat Epidemiol; 31: 251-62. 